\title{
El papel de las redes sociales en la actividad económica: el caso de los restauradores de Lille
}

\author{
Fabien Elorie ${ }^{1}$ - Universidad de Lillel (Francia)
}

\begin{abstract}
Resumen
Este artículo se sitúa en una doble perspectiva de sociología económica y de análisis de redes sociales. El enfoque que en ella se aplica no es egocéntrico, es decir, centrado en el nivel individual, sino que se trata de una visión sociocéntrica, o lo que es lo mismo, centrada en el nivel meso-social, sus estructuras objetivas y los procesos que lo gobiernan. Nos apoyamos en un caso empírico, el de trescientos restauradores instalados en la metrópolis de Lille, al norte de Francia, que reconstituyen una red completa interorganizacional. Tras haber explicado el modo en el que elaboramos nuestra población de forma empírica, nos interesamos por este mercado local de la restauración, tanto desde el punto de vista de la interfaz de producción (White) como desde el punto de vista del campo (Bourdieu). Mostramos la forma en la que dichas estructuras, económica y social, se articulan con la estructura relacional, entendida como el sistema de intercambio de recursos sociales (Lazega). De este modo, esperamos plantear una visión heterodoxa del funcionamiento de un mercado.
\end{abstract}

Palabras clave: red completa, recursos sociales, relaciones interorganizacionales restaurantes.

\begin{abstract}
This article is rooted in two fields of research: economic sociology and network analysis. The network approach we develop here is not an ego-network approach based on the individual level of analysis, but is a socio-centric approach based on the meso-social level of analysis. We focus our attention on the objective structures that govern markets. We rely on the empirical case of three hundred restaurants' owners situated in the area of Lille in the North of France. Our population reconstitutes an inter-organisational complete network. First, we explain how we have empirically constructed this population. Second, we present this restaurant industry, on the one hand as an interface of production (White) and, on the other hand as a field (Bourdieu). Third, we show how these economic and social structures are connected with the relational structure. In our view, the relational structure relates to a social exchange system of social resources (Lazega). Finally, our approach suggests a heterodox conception of how is running a market.
\end{abstract}

Key words: Complete network, social resources, inter-organizational ties, restaurants.

\footnotetext{
${ }^{1}$ Universidad de Lillel eloire.fabien@wanadoo.fr. El presente estudio, realizado en el marco de una tesis CIFRE, forma parte del programa de investigación ORIO (Observatorio de redes intra e interorganizacionales) y está financiado por EDF R\&D. Se lleva a cabo bajo la dirección de Emmanuel Lazega con la colaboración de Sylvaine Nugier y Sébastien Fagnoni.
} 


\section{I ntroducción}

Desde los años 60, la "restauración de tipo tradicional"2 en Francia se enfrenta a la aparición y al desarrollo de, por una parte, la "restauración rápida", y por otra, los establecimientos de franquicias o cadenas, ubicados en los centros de las ciudades y en los centros comerciales de la periferia. Esta situación hace que los propietarios de los establecimientos autónomos, tiendan a acudir, de forma más o menos inevitable, a los productos llamados de "ensamblaje culinario", procedentes de la industria agroalimentaria. A través de la industrialización y la racionalización de la producción culinaria, nace una forma específica de competencia, en términos de costes y precios, que examina las especifidades de los conocimientos artesanales de los establecimientos gastronómicos para los cuales, el modelo económico radica en la idea de que "los costes tienen poca importancia, siempre y cuando se logre la calidad adecuada". De este modo, el mercado de la restauración tradicional tiende a polarizarse en torno a dos modelos ideales típicos de empresas, que por un lado, se destacan por su calidad, de tipo artesanal y artístico y por otro, de tipo industrial y funcional (Mériot, 2002), y que valoran dos formas de éxito, una simbólica y otra económica.

Hoy en día, la restauración sigue estando muy dominada por las pequeñas empresas autónomas (desde el punto de vista jurídico). En la metrópolis de Lille, al norte de Francia, en donde se lleva a cabo nuestro trabajo de campo, el número de empresas no ha dejado de aumentar con bastante regularidad, a un ritmo del $+5 \%$ por término medio cada año desde los años 80 . No obstante, estos resultados no deben ocultar un alto índice de renovación: cada año, en término medio, desaparece el $15 \%$ de las empresas, frente al nacimiento del $20 \%$ de empresas nuevas. Este contexto de incertidumbre (según la doctrina keynesiana) frente al futuro explica los motivos que incitan la inscripción de estos profesionales en un movimiento doble, a la vez individual y colectivo, de cara a una toma de posición duradera en el mercado, y de cara a la regulación del espacio comercial que juntos estructuran. Intentaremos entender este doble movimiento en una perspectiva de sociología económica neoestructuralista. Desde una posición completamente heterodoxa de cara a la economía mainstream, intentaremos demostrar que este mercado está menos gobernado por la racionalidad instrumental de agentes aislados, que por formas de racionalidad social de actores interdependientes.

\footnotetext{
${ }^{2}$ Conforme con la definición de la NAF (nomenclatura de las actividades francesas), por oposición a la "restauración rápida".
} 
El estudio, cuyos resultados serán expuestos en el presente artículo, se llevará a cabo en una población de unos trescientos restauradores. La originalidad de la visión neoestructuralista en sociología (Lazega, Mounier, 2002; Lazega, 2008) radica en el hecho de que se esfuerza en reconstituir los sistemas de interdependencias relacionales entre actores de un mismo conjunto social. Negándose a considerar la red como un objeto de estudio por y para sí mismo, es decir, como una "forma de organización de la acción colectiva intermediaria entre el mercado y la organización" (Lazega, 1998, p.5-6), se esfuerza más bien en analizar los vínculos establecidos entre la estructura de una red y las demás estructuras sociales, económicas, etc. Esto implica la consideración de una multitud de definiciones de la noción de "relación social" y explica la innovación de los trabajos de White (acerca de la interfaz de mercado; 1981, 1992, 2002) y de Bourdieu (acerca del campo económico; 1997, 2000). Sobre todo, la teoría neoestructuralista hace hincapié en la existencia de dos fenómenos, que se pueden entender de forma empírica a nivel del análisis meso-social (entre el micro y el macro), que son la construcción de nichos sociales y la competición de estatus. De estos fenómenos se desprende la existencia de procesos sociales genéricos, como el control social, la regulación, la solidaridad limitada, el aprendizaje, etc., que son indispensables para los actores de la sociedad organizacional contemporánea.

Tras haber presentado, en una primera parte, nuestra metodología de estudio adaptada al nivel de análisis interorganizacional, presentaremos en la segunda, la construcción social de la estructura económica del mercado de la restauración de Lille, y terminaremos, en la tercera parte, con un análisis del fenómeno de construcción de nichos sociales que hace referencia al funcionamiento del proceso de solidaridad limitada.

\section{Metodología del estudio}

Para comprender el medio de la restauración de Lille, hemos realizado dos estudios de campo, uno cualitativo y exploratorio y otro cuantitativo mediante un cuestionario. El estudio exploratorio (abril-octubre de 2005) consistió en una decena de entrevistas semidirectivas con restauradores con perfil variado (desde los bares-restaurantes de barrio hasta los restaurantes gastronómicos de varios tenedores). El objetivo consistía en localizar la existencia de relaciones interorganizacionales mantenidas entre los establecimientos de restauración, es decir, la existencia de recursos sociales que puedan circular entre los restauradores. El estudio cuantitativo (de febrero a julio de 2006) consistió en la realización de un cuestionario cumplimentado directamente ante unos trescientos 
restauradores autónomos instalados en la metrópolis de Lille. Dicho cuestionario incluía tres tipos de variables: los atributos de los propios restauradores (carrera profesional, compromiso asociativo, características sociodemográficas, etc.); los atributos de sus establecimientos (tamaño, cantidad de empleados, precios aplicados, tipo de cocina, etc.); y las relaciones mantenidas entre dichos restauradores, relaciones que mediante nuestro estudio exploratorio podremos desglosar en distintos recursos sociales. En este artículo, hemos localizado principalmente dos de ellos: las conversaciones sobre la coyuntura por una parte y los intercambios de información útil por otra.

\subsection{Los recursos sociales}

Las conversaciones sobre la coyuntura son discusiones informales, diarias, que tienen los restauradores que se conocen al encontrarse: "Hablamos de nuestros volúmenes de negocios, de la coyuntura, los sueldos, de todo eso..." [TGR28]. Este recurso puede interpretarse, en la perspectiva de White, como uno de los medios mediante los cuales, en una interfaz de mercado, los productores "se observan entre sí"3 para adoptar una posición duradera. Así pues, estas discusiones son de gran utilidad para los restauradores, con vistas a entender mejor las fluctuaciones de su clientela (por ejemplo, si se detecta una caída del volumen de negocios, los restauradores la conciben de forma distinta en función de si sus colegas también afirman registrarla o no).

Los intercambios de información útil, sobre el personal, los proveedores, la cocina, los productos, las cuestiones jurídicas, etc., son igualmente informales, pero están impulsados por un espíritu de cooperación, ayuda mutua, asesoramiento mutuo4: "Mi vecino me dio algunos consejos hace algún tiempo, (...) por lo tanto, yo se lo devuelvo en función de los buenos oportunidades con los que cuente" [C3R92]. Este recurso puede interpretarse, en la perspectiva de la visión sociológica neoestructuralista, como un indicio de la posible presencia de nichos sociales que se elaboran y mantienen colectivamente, es decir, subgrupos de actores que mantienen "relaciones especialmente densas, multifuncionales, duraderas y vinculadas, directa o indirectamente con las actividades de producción". En el

\footnotetext{
3 También se interesan por la información incluida en las guías de restaurantes locales (Petit Futé, Chti, Oficina de turismo, etc.)

${ }^{4}$ Uzzi (1997) habla de una "fine-grained information transfer" (transmisión de información pertinente) que favorece la implantación de estrategias de empresas y el aumento de los márgenes gracias a los "planes adecuados". En nuestro estudio, este recurso está vinculado con el estatuto de "amigo", que los restauradores atribuyen por lo general a los colaboradores con los que afirman intercambiar información de utilidad.
} 
mundo económico, estos nichos sociales se interpretan como espacios en los que los actores se permiten "interrumpir parcial y temporalmente, los comportamientos puramente calculadores" (Lazega, Mounier, 2002, p.318).

\subsection{La construcción de la población}

Desde el punto de vista metodológico, existen dos vías para diseñar un análisis de redes sociales: el denominado enfoque egocéntrico, centrado en el individuo, el capital social individual; y el enfoque denominado sociocéntrico, centrado en el grupo social, el capital social colectivo (Laumann, Marsden, Prensky, 1983; Marsden, 1990). Nos ubicamos en la segunda visión, que se esfuerza en reconstituir los sistemas de interdependencias entre actores. Ésta exige la reconstitución de una red denominada (efectivamente, de forma abusiva) completa. En el marco de la visión sociocéntrica, la red completa se refiere a "un conjunto definido de actores" (Lazega, 1998, p.5), lo que implica la búsqueda de fronteras, y exige la creación de límites, en el conjunto social estudiado. Esta operación encuentra su coherencia a nivel meso-social, en donde se desarrolla este tipo de análisis de redes.

Debido al carácter potencialmente infinito de la red social interorganizacional de los restauradores de Lille, hemos intentado establecer distintos criterios de selección, para escoger entre los numerosos espacios y ramificaciones que la componen. La metrópolis de Lille, en donde realizamos nuestro estudio, cuenta con unos mil ochocientos restauradores autónomos. Nuestra población final incluye a trescientos: Por lo tanto, se trata efectivamente de una subpoblación, que carece no obstante de un estatus de muestra representativa en el sentido "estadístico"; dicha subpoblación es "teórica", es decir, está fundada en el principio de la "saturación", según la expresión utilizada por Glaser y Strauss (1967). Varios criterios priman simultáneamente en la construcción de nuestras fronteras empíricas: el criterio geográfico, es decir, la metrópolis de Lille, y algunas zonas (calles, barrios), elegidas como puntos de inicio de nuestro estudio de campo; el criterio institucional, es decir, vinculado con la definición inicial de nuestro objeto de estudios (restauradores tradicionales, autónomos desde el punto de vista jurídico, centrándonos principalmente en la cocina de tipo francesa, en su sentido amplio) y el criterio relacional, es decir, vinculado con la implantación de un proceso de selección iterativo: cada restaurador que pese a no pertenecer aún a nuestra lista, haya sido citado por otro restaurador, se integra en ella y se le entrevista. La gran dificultad del estudio radicaba en nuestra capacidad para cerrar el proceso. La experiencia demuestra que lo conseguimos, a pesar de que el cierre no sea nunca 
perfecto, y de que esté afectado obligatoriamente por las ausencias de respuestas en nuestro cuestionario, que representan aproximadamente un $10 \%$.

Este tipo de subpoblación "teórica" y "no representativa" nos impide utilizar nuestros resultados desde un punto de vista descriptivo: por ejemplo, los restaurantes gastronómicos están demasiado presentes. Sin embargo, esta decisión está vinculada con nuestro objeto de estudio, centrado en la reconstitución de "procesos universales" (Becker, 2002, p.148) en lugar de centrarse en las "cuestiones de distribución dentro de una población global".

\section{I nterfaz de mercado e interacciones}

La sociología económica cuenta con herramientas metodológicas para representar un espacio de mercado a partir de datos empíricos: el modelo $\mathrm{W}(\mathrm{y})$ de White es una de ellas. Para White, un mercado formado por productores es una interfaz. A diferencia del mercado neoclásico, que tan sólo entiende su objeto a partir de dos caras (la oferta y la demanda) y una única perspectiva (el encuentro de esta oferta con esta demanda), White considera que "cada mercado es tripartito (proveedores, productores, compradores), concepción que atribuye a una interfaz dos tipos de orientaciones posibles: una orientación upstream hacia los proveedores y una orientación downstream hacia los consumidores" (2002, p.11). Esta doble orientación implica que los productores son "equivalentes regulares" (Lazega, 1998, p.72) al estar relacionados por una parte, con el mismo tipo de proveedores y por otra, con el mismo tipo de clientela. Además, este concepto funda, en el pensamiento de White, la idea que defiende que los productores son interdependientes: "se observan entre sí".

Individualmente, cada uno busca un "nicho de calidad", es decir, una posición relativamente no competitiva en la estructura de mercado. Conjuntamente, todos participan juntos en la construcción de una escala de calidad, ajustando su producción respecto a los demás. A la vez competidores y colegas, los productores interdependientes garantizan juntos la viabilidad (o la ausencia de viabilidad) de la interfaz de mercado, mediante la coordinación de sus decisiones individuales.

\subsection{El modelo de White}


El modelo matemático elaborado por White ofrece una representación de la estructura de mercado mediante un plano dotado de dos dimensiones5 (véase la Figura 1). La primera dimensión tiene en cuenta el volumen de producción y la segunda, la calidad de la producción. Dentro de cada una de estas dimensiones, White tiene en cuenta, tanto los costes registrados por cada restaurador, como la satisfacción de la clientela en cuanto al nivel certificado. Las variables utilizadas para elaborar los ejes son: el número de clientes promedio por servicio, el valor de la factura promedio, la nota de calidad6, el número de empleados, el número de cubiertos, el número de servicios.

Una vez elaborado, el plano se divide en zonas en las que el mercado es "viable", es decir, que puede reproducirse, y "no viable", es decir, en situación de fracaso colectivo. las zonas "viables" se encuentran a su vez divididas en tres subzonas que forman una tipología de mercado. De este modo, cada establecimiento de restauración pertenece a un tipo de mercado, llamado paradójico, ordinario o avanzado, cada uno de ellos dotado de distintas características. Los restaurantes que pertenecen al tipo paradójico se caracterizan por una producción de "alta gama", una gran calidad y volúmenes de producción (relativamente) bajos. Por el contrario, los del tipo avanzado, se centran en los volúmenes producidos: cuanto mayores sean éstos, más bajos serán los costes debido a las economías de escala, pero la importancia acordada a la calidad es menor. El mercado de tipo ordinario aparece, por último, como una situación intermediaria: los costes de producción aumentan con la calidad, pero no obstante, la importancia relativa al volumen sigue siendo más fuerte que la relativa a la calidad (Steiner, 2005; Favereau, EymardDuvernay, Biencourt, 1994).

\footnotetext{
${ }^{5}$ Aquí no entraremos en las explicaciones de la construcción matemática del plano.

${ }^{6}$ Es decir, el reconocimiento otorgada por las guías gastronómicas francesas nacionales.
} 


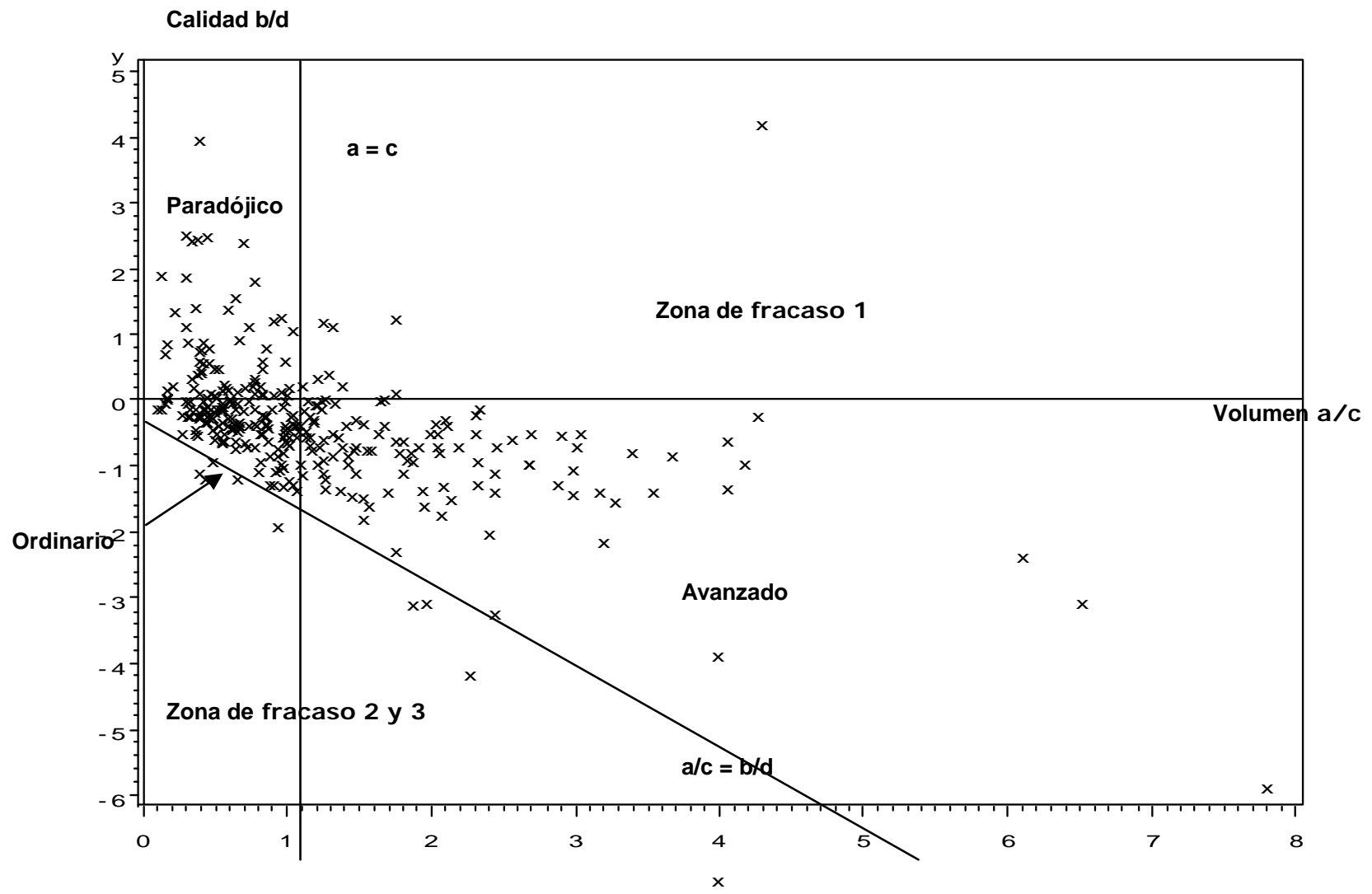

Figura 1. La interfaz de mercado a partir del modelo de W(y) de White ${ }^{7}$. Fuente: Eloire, 2007.

\subsection{La aplicación a los restauradores de Lille}

La aplicación a nuestros datos empíricos8 (véase la Figura 1) refleja que más del $85 \%$ de los establecimientos de nuestra población se encuentran ubicados en zonas llamadas "viables", con base al siguiente reparto: el mercado paradójico representa el $24 \%$ de los restauradores, el mercado ordinario, el $27 \%$ y el mercado avanzado el 34\%. Efectivamente, estas proporciones no son representativas del ámbito de actividad en su conjunto, pero debido a la variedad de la gama de establecimientos que incluye nuestra población, pensamos que añadir establecimientos suplementarios no deformaría este espacio (o lo haría de forma marginal), sino que consolidaría más bien la estructura.

Dicha visión de la estructura económica del mercado de los restauradores hace que la competición entre los mismos se considere dividida. Así pues, dentro de la interfaz, coexisten distintas formas de competición. En el mercado avanzado, la

\footnotetext{
${ }^{7}$ Agradezco a Thomas Dallery, por su gran ayuda en la realización de este gráfico.

${ }^{8}$ Los cuatro parámetros utilizados para elaborar los ejes son: a = el número de clientes promedio por servicio; $b=$ el valor del recibo promedio + la nota de calidad; $c=$ el número de empleados, el número de cubiertos * el número de servicios; $\mathrm{d}=$ Índice de disponibilidad de los empleados con respecto a los clientes.
} 
competición se lleva a cabo principalmente en los precios, es decir, mediante la capacidad a la hora de producir con menos costes que sus competidores: nos acercamos más a la teoría neoclásica. En el mercado paradójico, la competición se lleva a cabo mediante la calidad, es decir, mediante la capacidad a la hora de darse a conocer (por los homólogos y los clientes) y de resultar legítimo al fijar precios altos: aquí nos acercamos más a la economía de la calidad descrita, por ejemplo, por Karpik $(1989,2000,2007)$ y que necesita todo tipo de dispositivos de mediación (guías gastronómicas, revistas especializadas, apelaciones, denominaciones, certificaciones, etc.) y de instituciones (asociaciones profesionales, sindicatos), con vistas a elaborar colectivamente las opiniones y las clasificaciones de los productores.

\subsection{Los restauradores se observan entre sí}

En una interfaz, además de estar organizada en distintos tipos de mercados relativamente no competitivos entre sí, White también sugiere que para determinar su nicho de calidad individual y adoptar sus decisiones en cuanto a la atribución de los costes (relación entre calidad y volumen), los productores "se observan entre sí". Para comprobar este postulado relativo a la articulación entre interfaz y redes sociales, nos interesamos por las lógicas que respaldan las elecciones relacionales de los restauradores. Nuestros análisis abarcan las redes de discusiones y de información.

\section{Centralidades}

La primera constatación se refiere a los resultados de centralidad promedio (degree, indegree, outdegree) de los restauradores en función del tipo de mercado al que pertenecen (véase el Tabla 1). Constatamos que los restauradores ubicados en el mercado paradójico están, por término medio, mejor integrados en las redes, ya sea en términos de elecciones emitidas (outdegree) o de elecciones recibidas (indegree). Sin embargo, las diferencias son pocas entre los restauradores del mercado avanzado y los del mercado ordinario. Aquí se observa el efecto tan directo de la reputación. Así pues, los restauradores del mercado paradójico, es decir, del espacio de alta gama y gastronómico del mercado, no son sólo reconocidos por sus cualidades culinarias, sino que también son muy conocidos y muchos restauradores del mercado en general (incluyendo el paradójico) afirman intercambiar recursos sociales con ellos. La actividad relacional aparece como un componente de la actividad gastronómica, y como una manifestación que demuestra que la calidad es el fruto de una "construcción social" (White, 1981, 
2002). La calidad culinaria no sólo depende de una forma de capital cultural específica de la restauración vinculada con el diploma9 y con la experiencia profesional, sino que también depende de las redes de relaciones entre interconocidos e inter-reconocidos, y por lo tanto, del capital social según indica Bourdieu (1980), redes informales e incluso más formales mediante, tal y como veremos a continuación, la pertenencia a asociaciones profesionales honoríficas.

\begin{tabular}{llllll}
\hline \multirow{2}{*}{ Indicador } & Tipo de mercado & Paradójico & Avanzado & Ordinario & Promedio general \\
\cline { 1 - 2 } $\begin{array}{lllll}\text { Red de } \\
\text { discusiones }\end{array}$ & Degree & 40 & 12,5 & 13 & 21 \\
& Indegree & 21 & 6 & 6,5 & 11 \\
\multirow{2}{*}{$\begin{array}{l}\text { Red de } \\
\text { información }\end{array}$} & Outdegree & 19 & 6,5 & 6,5 & 10,5 \\
& Degree & 10 & 4 & 4 & 5,5 \\
& Indegree & 5 & 2 & 2 & 3 \\
\hline
\end{tabular}

Tabla 1. Centralidad, intensidad, asimetría de las redes sociales en función de los tipos de mercado. Fuente: Eloire, 2008.

Homofilia diádica

Tras haber señalado las diferencias de inversión relacional entre un tipo de mercado y otro, intentaremos identificar los fenómenos de homofilia, de tipo "Dios los cría y ellos se juntan", que pueden surgir en la interfaz. Haciendo referencia a White, planteamos la hipótesis de que los restauradores tienden principalmente a elegir sus relaciones entre sus colegas/competidores cercanos en la estructura de la interfaz, es decir, pertenecientes al mismo tipo de mercado que ellos. Para ello, utilizamos el programa Statnet10, cuyas capacidades de modelización estadística permiten realizar análisis de tipo ERGM11. Estos modelos permiten detectar la presencia de las subestructuras de tipo diádico y triádico en las distintas redes y probar su importancia. El fenómeno de homofilia da lugar primeramente a un conjunto de probabilidades en las díadas (ya sean recíprocas o no). De este modo, cada vez que se observa una relación entre dos restauradores, el parámetro de homofilia prueba la probabilidad de que ambos restauradores pertenezcan al mismo tipo de mercado.

\footnotetext{
${ }^{9}$ En Francia, no se exige diploma alguno para poder ejercer la profesión de restaurador.

${ }^{10} \mathrm{http}: / /$ csde.washington.edu/statnet/

${ }^{11}$ Exponential random graph models (modelos gráficos aleatorios exponenciales).
} 
Los resultados de los modelos ERGM12 presentan parámetros que, en las dos redes estudiadas, son significativos y positivos, salvo uno de ellos (el del mercado ordinario en la red de información), que es negativo (véase el Tabla 2). Así pues, este resultado confirma nuestra hipótesis inicial y se inscribe en el enfoque de White. En la red de discusiones, los restauradores tienden a discutir entre ellos dentro de cada tipo de mercado. En la red de información, los resultados resultan más matizados: en el mercado paradójico, el efecto de homofilia es el más importante, los "buenos oportunidades" y los "buenos negocios" son muy fiables y pertinentes al proceder de restauradores que gozan de la misma orientación culinaria; la misma constatación se refleja en el mercado avanzado, donde el efecto sigue siendo positivo, pero no obstante es más débil; el sentido negativo del parámetro de homofilia en la red de información, para el mercado ordinario, es interesante ya que los resultados parecen reflejar la posición intermediaria de dichos restauradores entre dos modelos de restauración (gastronómico o funcional) y podemos pensar que estos últimos, en función de su sensibilidad o su ambición, intentan establecer principalmente contactos e intercambiar información, no con sus semejantes, sino con restauradores que representan para ellos, arquetipos del modelo ideal.

\begin{tabular}{lcc}
\hline & Red de discusiones & Red de información \\
Mercado paradójico & $+0,1429(>0,01)$ & $+0.4148(>0,01)$ \\
Mercado ordinario & $+0,1419(>0,01)$ & $-0.0312(>0,01)$ \\
Mercado avanzado & $+0,1814(>0,01)$ & $+0.0556(>0,01)$ \\
\hline
\end{tabular}

Tabla 2. Homofilia diádica en las redes de discusiones y de información en función del tipo de mercado. Fuente: Eloire, 2007; Programa Statnet.

Homofilia triádica

En línea con la misma lógica, probamos en nuestros modelos ERGM un segundo parámetro, triádico esta vez, que representa el ciclo, o el intercambio generalizado según la definición acordada por Lévi-Strauss a este concepto13. Mientras que el parámetro de homofilia diádico que hemos probado anteriormente no incluye ningún efecto de reciprocidad, el del intercambio generalizado puede entenderse

\footnotetext{
12 Un modelo por red.

13 "J unto y además del intercambio entendido en su sentido limitado, es decir, cuando dos colaboradores intervienen exclusivamente, podemos identificar, y existe, un ciclo menos perceptible de forma inmediata, precisamente porque utiliza una estructura de mayor complejidad; a este ciclo lo llamamos intercambio generalizado" (1967, p.271).
} 
como una forma de reciprocidad indirecta: A da a B, que a su vez da a C; sin embargo, A no recibirá obligatoriamente y directamente de $B$, pero quizás reciba de C. Para que la reciprocidad indirecta funcione, cada individuo debe poder estar seguro (aunque no sea consciente de ello obligatoriamente) de que otro individuo de la red (que no deberá ser obligatoriamente el interlocutor directo) terminará por "devolverle el favor". Desde un punto de vista sociológico, la presencia de este parámetro puede ilustrar un proceso de solidaridad limitada en el conjunto social.

Por lo general, dentro de nuestras redes de discusiones y de información, este parámetro triádico es significativo y negativo: no hay solidaridad entre los restauradores por lo general. Cuando introducimos la pertenencia a un tipo de mercado14, todos los parámetros son significativos y negativos, salvo uno de ellos, el del mercado paradójico, que es positivo (véase el Tabla 3). Por lo tanto, estos resultados no confirman nuestra hipótesis tal y como se indicó anteriormente. El efecto negativo del parámetro de intercambio generalizado desvela ante todo el carácter estratégico de los intercambios de recursos entre colegas/competidores: por una parte, la limitación de reciprocidad directa es fuerte, y ésta aparece como el motor de la circulación de los recursos de discusiones e información, y por otra, a pesar de que las relaciones tiendan a ser homófilas en cada tipo de mercado, el intercambio indirecto parece estar prohibido. En cuanto al carácter positivo del parámetro de intercambio generalizado para el mercado paradójico en la red de discusiones, éste constituye otra manifestación del fenómeno de construcción social de la calidad, expresando la importancia del desarrollo de las redes sociales informales, pero también, de la existencia de varias asociaciones, formalmente constituidas, que favorecen a la vez las reuniones, el inter-reconocimiento y el establecimiento de una base de valores y prácticas comunes.

\begin{tabular}{lll}
\hline & Red de discusiones & Red de información \\
Mercado paradójico & $+0,2549(>0,01)$ & $-0.1275(>0,01)$ \\
Mercado ordinario & $-0,3054(>0,01)$ & $-0.3681(>0,01)$ \\
Mercado avanzado & $-0,2565(>0,01)$ & $-0.2914(>0,01)$ \\
\hline
\end{tabular}

Tabla 3. Intercambio generalizado en las redes de discusiones y de información en función del tipo de mercado. Fuente: Eloire, 2007; Programa Statnet.

\footnotetext{
14 Esto prueba la existencia de una homofilia triádica: cuando existe un ciclo en una red, ¿esto quiere decir que los restauradores pertenecen al mismo tipo de mercado?
} 


\section{Campo y nichos sociales}

Como espacio de producción y de intercambio comercial y no comercial, una interfaz de mercado también constituye un campo en el cual actúan fuerzas sociales y simbólicas, objetivadas bajo la forma de distintos tipos de capital. De este modo, el campo de la restauración de Lille se estructura en torno a cuatro grandes dimensiones socio-económicas: el capital económico15, el capital cultural específico vinculado con las destrezas culinarias, el capital de reconocimiento social gastronómico y el capital organizacional (Eloire, Fagnoni, 2008).

En términos metodológicos y estadísticos, un campo consiste en un análisis de correspondencias múltiples (ACM) en un juego de variables cualitativas, o cuantitativas agrupadas por clases, que permite tener en cuenta, de forma simultánea, todas las variables de nuestro cuestionario.

Aquí, la unidad de análisis yace en el restaurador, individualizado mediante sus atributos objetivos; pero a cada uno de ellos también se vinculan características organizacionales, que los inscriben en colectivos, ya sea laborales (su restaurante) o de otro tipo (los sindicatos, las asociaciones comerciales u honoríficas). Aunque las propiedades individuales hagan referencia a disposiciones fijas y fundadoras de los habitus, las características organizacionales se refieren a las posiciones y tomas de posición individuales, actuales o potenciales (en el espacio de las posibilidades) dentro de la estructura del interfaz o del campo: estilo de cocina, distinciones y recompensas obtenidas, tipos de afiliaciones profesionales, compromiso sindical, todas ellas constituyen las tomas de posiciones específicas en el ámbito de la restauración.

\subsection{El campo de la restauración de Lille}

A nivel meso-social, el campo de la restauración de Lille (véase la Figura 2), recuerda la estructura del espacio social global (Bourdieu, 1979, 1994). El eje horizontal (11,9\% de la información de la ACM) describe una "estructura cruciforme": una relación simétrica e inversa tiende a establecerse entre el capital económico, vinculado con el tamaño de la empresa, y el capital culinario, vinculado con los diplomas, experiencias y destreza del restaurador, de forma que cuanto mayor sea el capital económico, menor será el capital culinario, y viceversa, cuanto mayor sea el capital culinario, menor será el capital económico. El eje vertical

\footnotetext{
${ }^{15}$ Aquí retomamos las variables utilizadas para la construcción de la interfaz.
} 
$(5,7 \%)$ ubica a los restauradores en función del volumen de su capital global. Tres formas de capital, de las cuatro señaladas, contribuyen a determinar este volumen de capital global: el capital económico, el capital (cultural) culinario y el capital (social) gastronómico, vinculado con las recompensas obtenidas en las guías francesas como la de Michelín.

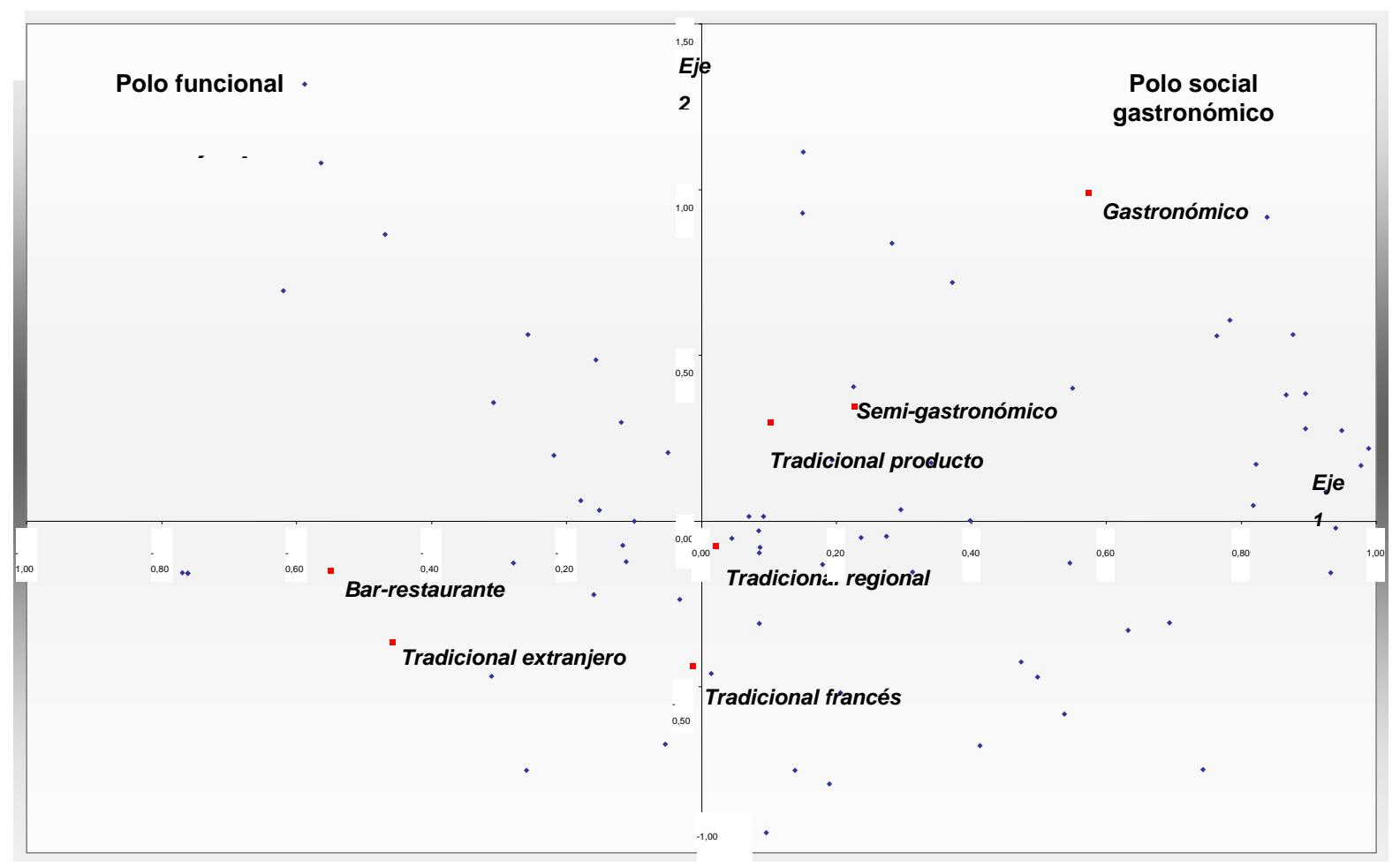

Figura 2. Jerarquía de estilos y especialidades culinarias. Fuente: Eloire, 2008.

Al proyectar como variable suplementaria en la ACM los estilos de cocina, constatamos que las posiciones en el campo (según la estructura y el volumen de capital) determinan las tomas de posición culinarias: la cocina gastronómica la practican los productores que concentran a la vez, el capital culinario y gastronómico; la cocina tradicional la ejercen aquéllos que no cuentan con ninguna forma de capital (culinario, económico o gastronómico); y por último, la cocina de bar-restaurante la adoptan aquellos restauradores poco dotados de capital culinario, que están mejor dotados de capital económico16. Principalmente, el

\footnotetext{
16 Más concretamente, la gastronomía (es decir, la cocina de alta gama) se desglosa en cocina gastronómica y semi-gastronómica; la cocina tradicional (es decir, la cocina de gama intermedia) reúne especialidades muy diversas, que hemos agrupado del siguiente modo: cocina tradicional francesa (todos los tipos de platos y recetas francesas); cocina tradicional regional (platos con connotación regional); cocina tradicional de producto (cocina especializada en un producto o en un concepto en concreto);
} 
campo ordena dichos estilos en función de las posiciones de los establecimientos y de las disposiciones de los restauradores. Así pues, se establece una jerarquía: en la cima de la clasificación, en el lado del polo gastronómico (ubicado en la parte superior derecha) encontramos la cocina gastronómica; a continuación, la cocina semi-gastronómica y la cocina tradicional por producto, cuyo ejercicio parece exigir, por parte de los restauradores, un volumen de capital más alto que la cocina tradicional regional y la cocina tradicional francesa. Los restauradores que practican una cocina tradicional regional tienden a estar mejor dotados de capital global que los que practican una cocina tradicional francesa. Las cocinas de bar-restaurante y especialidades extranjeras son ajenas a esta jerarquía, ya que se ordenan del lado del polo económico y funcional, centrado en la dimensión del servicio ofrecido en lugar de centrarse en el carácter artístico (Mériot, 2002). Esta doble polaridad que caracteriza el campo de la restauración (funcional y gastronómica) hace referencia a las dos dimensiones genéricas que entran en la construcción del interfaz (volumen y calidad); por otra parte, los tipos de mercado pueden interpretarse en función de los estilos culinarios.

\subsection{La red de afiliaciones de los restauradores de Lille}

Las formas de estatus social y económico, procedentes de la estructura y del volumen de capital de los restauradores y de sus tomas de posiciones individuales adoptadas en materia culinaria, se institucionalizan mediante la aparición de distintos tipos de asociaciones oficialmente constituidas, entre las cuales distinguimos, los sindicatos profesionales (como la UMIH o Eurotoques), las asociaciones con fines comerciales (como Hourra Gand o Lille Events) y las comunidades de jefes de cocina, que surgen a distintos niveles, desde el nivel local (como las Tables Gourmandes, formada por unos treinta jefes de cocina que desean promover la gastronomía regional) hasta el nivel nacional (como los Disciples d'Escoffier, Prosper Montagné, les Maîtres cuisiniers de France, Générations.C). La existencia de estas asociaciones formales muestra que la acción colectiva existe entre los competidores (véase la Figura 3 ).

El estudio sociológico de las asociaciones profesionales es particularmente interesante, ya que éstas desarrollan prácticas de selección de sus miembros, que objetivan las jerarquías sociales señaladas por el campo. En las asociaciones con fines comerciales, la pertenencia a un mismo sector geográfico o a una misma 
gama determina la adhesión. En las asociaciones con ánimo honorífico, la adhesión es individual y voluntaria, pero está sujeta a la aprobación de los demás miembros de la organización: se elaboran normas de cooptación de los nuevos miembros para garantizar la "calidad" social y profesional de los que entran. Los trámites necesarios son más o menos idénticos en todos los casos: hay que demostrar que se cuenta con una "sólida experiencia profesional" (que incluso puede exigir la cumplimentación de un "cuestionario sujeto a la validación de una comisión de investigación"), y ser apadrinado por dos miembros de la asociación, condición que refleja por sí misma la importancia del estudio de las redes sociales en los medios profesionales.

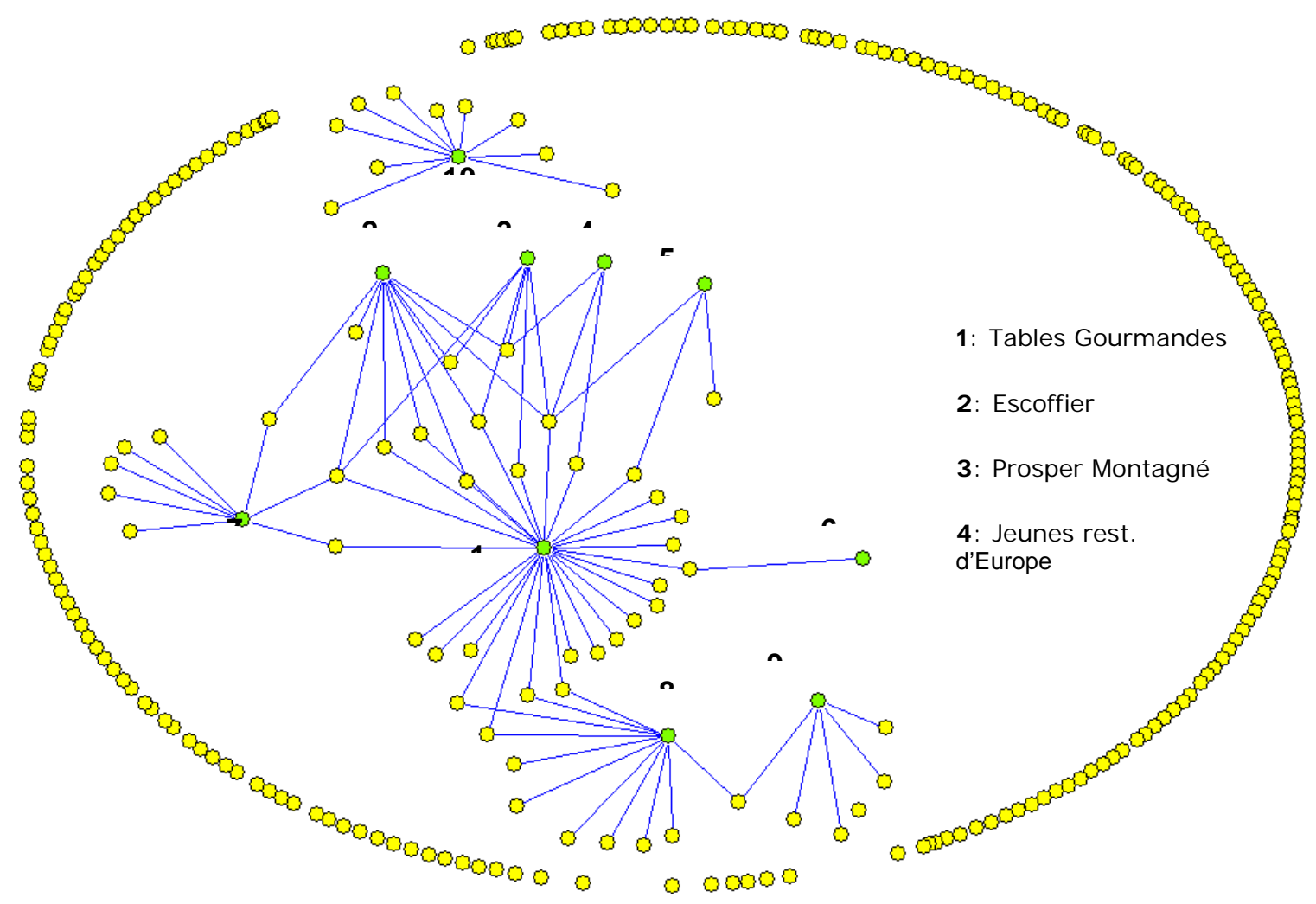

* Miembros activos exclusivamente.

Figura 3. Red de afiliaciones en el mercado de la restauración de Lille. Fuente: Eloire, 2008. Realizado con el programa Pajek.

Estas afiliaciones, principalmente a las asociaciones honoríficas, conllevan grandes significados sociales y profesionales. Constituyen un vector esencial de la construcción de la profesión, y dentro de la profesión, de la "calidad" de los 
productos y de sus productores. No obstante, transmiten concepciones distintas de la gastronomía, al proponerse, por ejemplo, "conservar y transmitir la gran herencia del arte culinario", o "marcar las nuevas vías de la cocina francesa".

Desde el punto de vista del análisis de las redes sociales, los vínculos de afiliación nos permiten trazar una red "dual" de relaciones de pertenencia a "colectivos sociales importantes", mediante las cuales "las relaciones interpersonales se convierten en relaciones interinstitucionales" (Pizarro, 1999, 2007). Sin embargo, en el caso de los restauradores de Lille, esta red dual tan sólo afecta a unos sesenta restauradores incluidos en nuestra población. Por otra parte, el $50 \%$ de ellos proceden del mercado paradójico; y esta proporción asciende al $70 \%$ al considerar exclusivamente las afiliaciones a asociaciones de tipo honorífico. Esta constatación se une a lo indicado anteriormente acerca de la importancia de la inversión relacional en la construcción social de la calidad: la acción colectiva está organizada y respaldada principalmente (aunque no de forma exclusiva) por restauradores de alta gama.

\subsection{Procesos de solidaridad limitada entre competidores}

El mercado de la restauración no está formado por productores completamente aislados que pretenden conseguir exclusivamente sus propios objetivos, como lo indica la teoría económica neoclásica. Se manifiestan lógicas colectivas profesionales, pero en ellas, cada restaurador se compromete, de forma individual, ante niveles distintos (véase el Tabla 1) y en función de distintas modalidades. La participación en la competición de estatus económico o gastronómico, la pertenencia a colectivos formalmente organizados para la defensa de intereses (en su sentido más amplio) de la profesión, hace que los restauradores pongan en marcha relaciones interindividuales de transmisión e intercambio informal de recursos sociales. Tras haber indicado anteriormente los fenómenos de homofilia y reciprocidad diádica y triádica que los guían, ahora estudiaremos el fenómeno de construcción de nichos sociales, subgrupos informales en los cuales algunos restauradores pueden cooperar. 

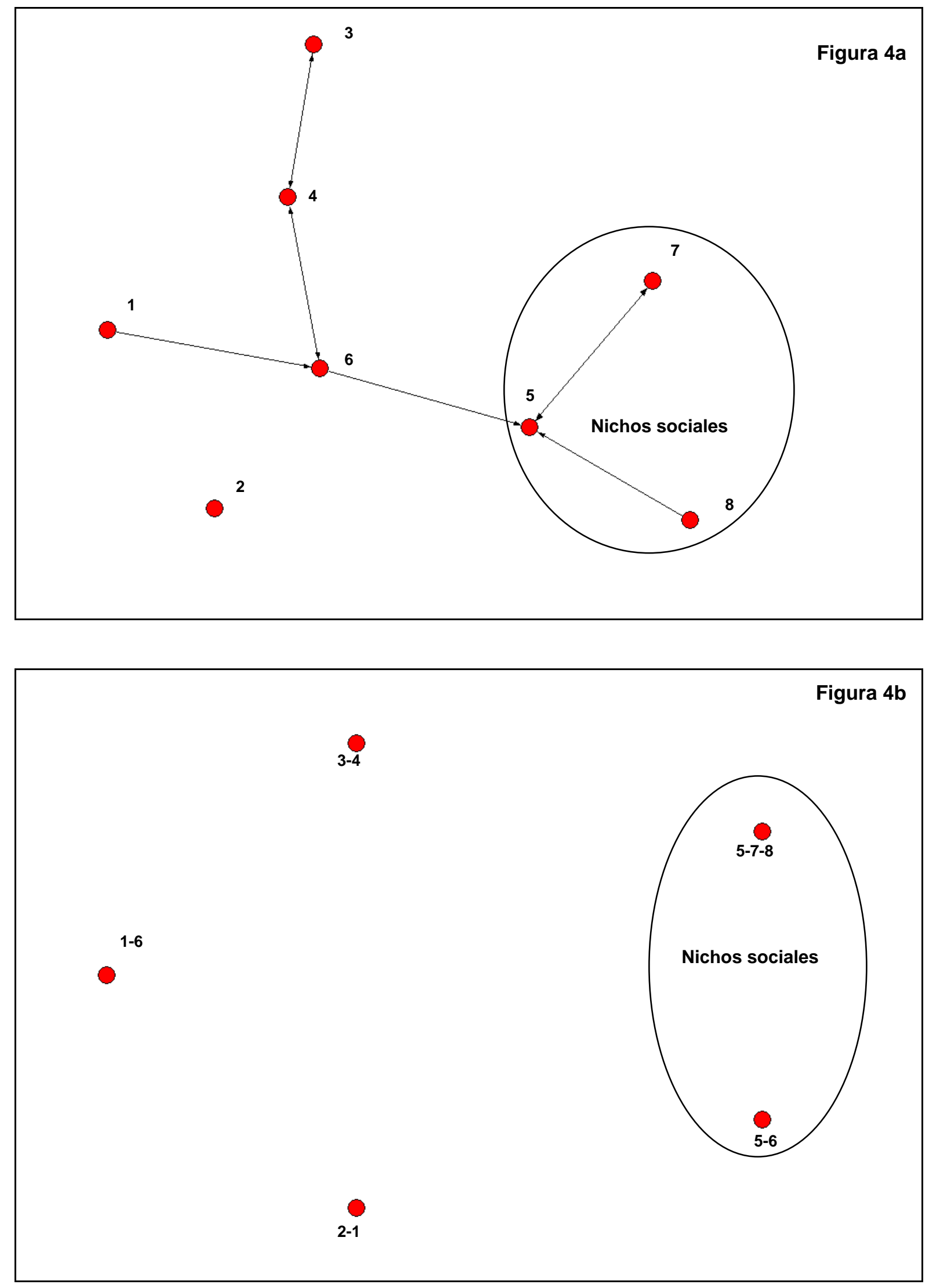

Figura 4. Comparación de los nichos sociales en blockmodels de discusiones (4a) y de información (4b). Fuente: Eloire, 2008. 


\section{La identificación de los nichos sociales}

La identificación de los nichos sociales en una red completa se lleva a cabo gracias al blockmodel ${ }^{17}$ (White, Boorman, Breiger, 1976), fundado en la idea de equivalencia estructural (Lorrain, White, 1971). Esta herramienta estadística permite principalmente reflexionar sobre la noción de grupo en el ámbito de la sociología: dos actores estructuralmente equivalentes tienen las mismas relaciones con las mismas personas. Sin embargo, esto no implica que éstos interactúen juntos. De este modo, el grupo social se define de dos formas. Primeramente, mediante las relaciones internas al grupo (densidad intra-grupo): ¿sus miembros tienen relaciones entre sí? En segundo lugar, mediante las relaciones ajenas al grupo (densidad inter-grupo): ¿con los miembros de qué otro(s) grupo(s) suelen tener relaciones? Realizamos este análisis en las dos redes estudiadas aquí: las conversaciones sobre la coyuntura y los intercambios de información. Obtenemos dos estructuras relacionales muy distintas (véase la Figura 4): una formada por ocho bloques, algunos de los cuales se encuentran relacionados entre sí, y la otra formada por cinco bloques no relacionados. Todos los bloques no tienen el estatus de nicho social (o sub-grupo): tan sólo lo son aquéllos cuyas relaciones intra-grupo gozan de una densidad relativamente alta; los demás reúnen a restauradores que están más o menos atomizados, y cuyo único punto en común radica en sus relaciones con otros restauradores que pertenecen a bloques distintos.

En la red de discusiones, los nichos sociales son los bloques 5, 7 y 8 (véase la Figura 4a) y en la red de información, son los bloques 5-6, y 5-7-818 (véase la Figura 4b); por el contrario, los bloques 1 y 2-1 reúnen respectivamente a los restauradores aislados (aquéllos que carecen de relaciones de discusión y de información). Aquello que realmente ponen de manifiesto los blockmodels radica en la existencia de formas de solidaridad informales y limitadas dentro del mercado: informales, ya que los nichos sociales se desarrollan fuera de cualquier existencia jurídica y limitadas al fundarse en un principio de discriminación (intercambiar con algunos, conlleva evidentemente la exclusión de todos los demás). Esto es aún más visible en el blockmodel de información que en el de conversaciones, ya que sus bloques no están relacionados entre sí: es cierto que la información circula entre los competidores, pero esto surge principalmente dentro de los nichos sociales, y no entre ellos, o en su caso, de forma marginal.

\footnotetext{
${ }^{17}$ Realizado con el programa Ucinet: http://www.analytictech.com/ucinet/ucinet.htm
} 


\section{El análisis y la interpretación de los nichos sociales}

Los restauradores que forman los nichos sociales del blockmodel de conversaciones $(5,7,8)$ son aproximadamente los mismos que forman los nichos sociales del blockmodel de información (5-6; 5-7-8), a diferencia de que en el segundo, las configuraciones son distintas: esto está vinculado por una parte con la naturaleza del recurso intercambiado (los intercambios de información son más confidenciales y más sensibles19 que las conversaciones sobre la coyuntura) y por otra, con el fenómeno de competición de estatus. La descripción estadística de los atributos de los restauradores que forman cada nicho social refleja efectivamente que dichas construcciones relacionales no están desconectadas de la estructura social (campo) y económica (interfaz) del mercado de la restauración. Una vez más, volvemos a observar que los nichos sociales suelen tender a formarse entre los restauradores que comparten atributos vinculados con la calidad, es decir, que poseen un volumen más alto de capital culinario y de capital gastronómico que la media, lo cual consolida la existencia de los efectos de homofilia que hemos diagnosticado previamente. No obstante, esto no explica la aparición de dos nichos sociales distintos (5-6; 5-7-8), dentro del polo gastronómico del mercado.

A nivel del análisis se marca el vínculo entre las relaciones de afiliación y los recursos sociales, mediante una especie de superposición de lo informal y lo formal. Para ilustrar este fenómeno, hemos realizado un cuadro sencillo transversal, entre los vínculos de afiliaciones y los dos nichos sociales que compiten en el mercado gastronómico (véase el Tabla 4). La primera constatación abarca la distribución, en los nichos sociales, de los miembros de las Tables gourmandes (sombreados). Formado por unos treinta miembros, este club local de jefes de cocina desempeña un papel central y estructurante en la vida relacional de dicho medio profesional local (véase la Figura 3), al reunir a los principales jefes locales y al fijarse como objetivo el promover la gastronomía del norte. La tabla refleja que sus miembros proceden, a partes casi iguales, de los restauradores que forman los dos nichos sociales 5-6 y 5-7-8. Por lo tanto, pese a su homogeneidad aparente, este Club de jefes de cocina de Lille resulta un grupo heterogéneo. Este vínculo que une formalmente a los miembros de la asociación, no debe ocultar el entorno de

\footnotetext{
18 Los números de bloques en el blockmodel de información corresponden a los del blockmodel de discusiones.

19 Los intercambios de información están muy vinculados con las relaciones de amistad entre los restauradores, aspecto que no abordaremos en este estudio.
} 
competición de estatus en el que se encuentran, y que se observa al estudiar los intercambios informales de información.

\begin{tabular}{lccc}
\hline & $\begin{array}{c}\text { Nicho social 5- } \\
\mathbf{6}(\mathbf{n}=\mathbf{4 0})\end{array}$ & $\begin{array}{c}\text { Nicho social 5- } \\
\mathbf{7 - 8}(\mathbf{n}=\mathbf{4 0})\end{array}$ & $\begin{array}{c}\text { Otros bloques } \\
\mathbf{2 0 0}\end{array}$ \\
\hline Tables Gourmandes & 15 & 13 & 1 \\
Prosper Montagné & 5 & 1 & 0 \\
Disciples d'Escoffier & 9 & 1 & 0 \\
Générations.C & 3 & 0 & 0 \\
JRE & 3 & 0 & 0 \\
MCF & 1 & 0 & 0 \\
\hline
\end{tabular}

Tabla 4. Cuadro transversal de vínculos de afiliación y nichos sociales. Fuente: Eloire, 2008.

La segunda constatación nos permite profundizar el entendimiento del fenómeno de la competición de estatus. Cuando observamos la zona donde son contratados los restauradores que forman parte de asociaciones de tipo honorífico nacional, constatamos que todos o casi todos pertenecen al nicho social 5-6. De este modo, la diversidad de las asociaciones honoríficas, que transmiten ideas a menudo muy distintas de la gastronomía, no debe ocultar la complicidad objetiva de sus miembros en el intercambio de información. Estos restauradores, los más reconocidos, pertenecen al mismo nicho social, parecen considerarse objetiva y mutuamente como interlocutores legítimos pese a sus divergencias de puntos de vista sobre la gastronomía. Podemos interpretar esta forma de solidaridad limitada que muestran, como un medio para alimentar y conservar el estatus que poseen, excluyendo de sus intercambios a los miembros menos prestigiosos del segundo nicho social. En otras palabras, en la zona donde habíamos pensado detectar homogeneidad, es decir, en el Club de las Tables gourmandes, aparece en realidad una estructura formada por dos nichos sociales con estatus heterogéneo, y por el contrario, donde creíamos que se desarrollaban conflictos y tensiones, es decir, entre los miembros de asociaciones honoríficas que transmiten ideas distintas de la gastronomía, constatamos principalmente una cierta complicidad objetiva.

\section{Conclusión}

Para concluir esta investigación, nos gustaría hacer hincapié en tres puntos: la capacidad de la sociología económica para proponer una visión heterodoxa del mercado; la capacidad del análisis de las redes sociales para poner de manifiesto los procesos sociales esenciales para la vida social y económica; y el interés que 
representa la llamada visión sociocéntrica para la producción de conocimientos a nivel del análisis meso-social.

La sociología económica, que registra una renovación desde los años 70 (Gislain, Steiner, 1995; Steiner, 1999; Lévesque, Bourque y Forgues, 2001; Convert, Heilbron, 2004), se institucionaliza en torno a una serie de corrientes muy variadas, pero que tienen como punto en común el hecho de identificarse en la metáfora del embeddedness (Polanyi, 1944), que afirma que toda sociedad tiene una economía, y toda economía tan sólo puede desarrollarse en una sociedad. Este postulado legitima el estudio de las "estructuras sociales de la economía" (Bourdieu, 2000) y por lo tanto, el estudio de los mercados como "construcciones sociales" (White, 1981, 2002). Dentro de este amplio programa de investigación, inscribimos nuestro trabajo sobre los restauradores de Lille acerca de los que estudiamos tres tipos de relaciones sociales: las formas de capital, que ubican a los restauradores en un campo; las afiliaciones formales, que los ubican en el proceso de institucionalización de la profesión; y las transmisiones e intercambios de recursos sociales, que los ubican en un sistema relacional.

Describimos los vínculos existentes entre estas relaciones distintas, e intentamos demostrar que un mercado no está gobernado exclusivamente por los mecanismos de la competencia de precios, sino también mediante aquéllos de la competencia de calidad. El uso de las redes sociales como herramientas de investigación del mercado permite caracterizar mejor la naturaleza de estos fenómenos de competición. Los restauradores orientados hacia el polo gastronómico, principalmente, desarrollan una vida relacional, formal, a través de la creación de Clubes y sindicatos, e informal, mediante la creación de nichos sociales. De este modo, en este mercado describimos las formas adoptadas por el proceso social de solidaridad limitada, fundado en criterios de reciprocidad de los vínculos y de homofilia en términos de estatus y de estilo.

Estos análisis han sido posibles mediante la adopción de una visión que hace que nos centremos en el grupo, con un enfoque sociocéntrico en lugar de centrarnos en el individuo, según la visión egocéntrica. Este carácter sociocéntrico de nuestra población, su estatus de red completa interorganizacional, es el que permite aplicar a nuestros datos los conceptos de interfaz y de campo, y ofrecer de este modo un mejor conocimiento de los fenómenos sociológicos a nivel meso-social (entre lo micro y lo macro). A nuestro parecer, este nivel de análisis está concretamente adaptado a la sociedad organizacional contemporánea, es decir, una sociedad gobernada por "grandes máquinas organizacionales", de la que también forman 
parte los mercados, y cuyo funcionamiento "exige cantidades inéditas de coordinaciones estrechas entre miembros altamente interdependientes" (Lazega, 2008). Estos análisis del nivel meso intentan desvelar los procesos sociales que actualizan localmente las desigualdades a nivel macro, reflejando por ejemplo: el modo en que la competición de estatus y los nichos sociales informales permiten la formación de oligarquías locales (temporalmente) solidarias; las causas que explican que un mercado esté lejos de poner a todos los participantes al mismo nivel de igualdad, sino que elabora por sí mismo sus propias jerarquías y formas de estatus; y el modo en el que éste, lejos de autorregularse, para funcionar, necesita instituciones cuya principal finalidad sea la reducción de la incertidumbre de cara al futuro. Efectivamente, el hecho de considerar la incertidumbre (según la doctrina keynesiana) en pleno centro del mercado es lo que otorga a nuestro enfoque su carácter heterodoxo (Bessis, 2008; Lavoie, 2004; White, Godart, Corona, 2008).

\section{Referencias}

Becker, H.S. (2002). Les ficelles du métier, comment conduire sa recherche en sciences sociales. Guides Repères, La Découverte.

Bessis, F. (2008). "Quelques convergences remarquables entre I'Economie des Conventions et la Théorie de la Régulation", Revue Française de Socio-Economie, $n^{\circ} 1$, pp. 9-25.

Bourdieu, P. (1979). La distinction. Paris, Editions de Minuit.

Bourdieu, P. (1980). "Le capital social, notes provisoires", Actes de la Recherche en Sciences Sociales, $n^{\circ} 31$, p. 2.

Bourdieu, P. (1994). Raisons pratiques, sur la théorie de l'action. Paris, Seuil.

Bourdieu, P. (1997). "Le champ économique", Actes de la Recherche en Sciences Sociales, $n^{\circ} 119$, pp. 48-66.

Bourdieu, P. (2000). Les structures sociales de l'économie. Paris, Seuil.

Convert, B., Heilbron, J. (2004). "Genèse de la "nouvelle sociologie économique" aux Etats-Unis", in. Heilbron, J., Lenoir, R., Sapiro, G., Pour une histoire des sciences sociales, hommage à Pierre Bourdieu, Fayard.

Eloire, F., Fagnoni, S. (2008). "Classification de variables: une application pour la sociologie quantitative et l'analyse des réseaux sociaux", no publicado.

Favereau, O., Eymard-Duvernay, F., Biencourt, O. (2002). "Where do markets come from? From (quality) conventions!", in. Favereau, Lazega (eds), Conventions and Structures in Economic Organization: Markets, Networks, and Hierarchies, Cheltenham, Edward Elgar Publishing, pp. 213-252.

Gislain, J.J., Steiner, Ph. (1995). La sociologie économique, 1890-1920. Paris, PUF. 
Glaser, B.G., Strauss, A.L. (1967). The discovery of grounded theory: strategies for qualitative research. Adline Transaction.

Karpik, L. (1989). “L'économie de la qualité", Revue Française de Sociologie, 30, pp. 187-210.

Karpik, L. (2000). “Le Guide Rouge Michelin", Sociologie du Travail, 42, pp. 369389.

Karpik, L. (2007). L'économie des singularités, NRF, Gallimard.

Laumann, E.O., Marsden, P.V., Prensky, D. (1983). “The Boundary specification problem in network analysis", in Burt, R., Minor, M.J., Applied Network Analysis, Beverly Hills: Sage.

Lavoie, M. (2004). L'économie postkeynésienne. Éditions La Découverte, col. Repères.

Lazega, E. (1998 [2007]). Réseaux sociaux et structures relationnelles. Que sais-je, PUF.

Lazega, E., Mounier, L. (2002). "Interdependent entrepreneurs and the social discipline of their cooperation: The research program of structural economic sociology for a society of organizations", in Favereau, Lazega (eds), Conventions and Structures in Economic Organization: Markets, Networks, and Hierarchies. Cheltenham, Edward Elgar Publishing, pp. 147-199.

Lazega, E. (2008). "Théorie de la coopération entre concurrents : organisations, marchés et réseaux", in. Steiner et Vatin, Traité de sociologie économique, PUF (próxima publicación).

Levesque, B., Bourque, G., Forgues, E. (2001). La nouvelle sociologie économique: originalité et diversité des approches. Paris, Desclée de Brouwer.

Lévi-Strauss, C. (1967). Les structures élémentaires de la parenté. Paris, La Haye, Mouton Editeur, éd. La Maison des Sciences de l'Homme.

Lorrain, F., White, H.C. (1971), "Structural equivalence of individuals in social networks", Journal of Mathematical Sociology, $n^{\circ} 1$, pp. 49-80.

Marsden, P. V. (1990). "Network data and measurement", Annual Review of Sociology, 16, pp. 435-463.

Mériot, S.-A. (2002). Le cuisinier nostalgique, entre restaurant et cantine. Paris, CNRS Editions.

Pizarro, N. (1999). “Appartenances, places et réseaux de places: la reproduction des processus sociaux et la génération d'un espace homogène pour la définition des structures sociales", Sociologie et société, vol. 31, n¹, pp.143-161.

Pizarro, N. (2007). "Structural identity and equivalence of individuals in social networks, beyond duality", International Sociology, vol. 22 (6), pp. 767-792, 2007.

Polanyi, K. (1944 [2005]). La Grande Transformation: aux origines politiques et économiques de notre temps. Paris, NRF, Gallimard. 
Steiner, Ph. (2005). "Le marché selon la sociologie économique", Revue Européenne des Sciences Sociales, Tomo XLIII, n²132, pp. 31-64.

Uzzi, B. (1997). "Social structure and competition in interfirm networks: the paradox of embeddedness", Administrative science quarterly, 42, pp. 35-67.

White, H., Boorman, S., Breiger, R. (1976). "Social structure from multiple networks I. blockmodels of roles and positions", American Journal of Sociology, $\mathrm{n}^{\circ} 81$, pp. $730-870$.

White, H. (1981). "Where do markets come from?", American J ournal of Sociology, 87 (3), pp. 517-587.

White, H. (1992). Identity and Control: a structural theory of social action. Princeton, Princeton University Press.

White, H. (2002). Markets from networks. Socioeconomic models of production. Princeton, Princeton University Press.

White, H., Godart, F., Corona, V. (2008). “Mobilizing identities, uncertainty and control in strategy", Theory, Culture, and Society, vol. 24, n-8, pp.191-212. 